AperTO - Archivio Istituzionale Open Access dell'Università di Torino

\title{
CDNA-AFLP analysis reveals a set of new genes differentially expressed in cucumber root apexes in response to iron deficiency
}

\section{This is the author's manuscript}

Original Citation:

Availability:

This version is available http://hdl.handle.net/2318/1655376

since 2018-01-15T11:31:18Z

Published version:

DOI:10.1007/s10535-012-0050-1

Terms of use:

Open Access

Anyone can freely access the full text of works made available as "Open Access". Works made available under a Creative Commons license can be used according to the terms and conditions of said license. Use of all other works requires consent of the right holder (author or publisher) if not exempted from copyright protection by the applicable law. 


\section{IIIS AperTO}

UNIVERSITÀ

DEGLI STUDI

DI TORINO

This is the author's final version of the contribution published as:

Vigani G, Chittò A, De Nisi P, Zocchi G. cDNA-AFLP analysis reveals a set of new genes differentially expressed in cucumber root apexes in response to iron deficiency. Journal of Biologia Plantarum. . volume 56 fascicolo 3, anno 2012, pagg 502-508 11. Doi: 10.1007/s10535012-0050-1

The publisher's version is available at:

https://link.springer.com/article/10.1007/s10535-012-0050-1\#enumeration

When citing, please refer to the published version.

\section{Link to this full text:}

https://link.springer.com/content/pdf/10.1007\%2Fs10535-012-0050-1.pdf

This full text was downloaded from iris-Aperto: https://iris.unito.it/ 


\begin{abstract}
Cucumber (Cucumis sativus L.) is considered a model plant for the investigation of Fe deficiency responses, since it strongly exhibits typical Strategy I activities (i.e. Fe(III)-chelate reductase [FC-R], $\mathrm{H}^{+}$-ATPase and Iron Regulated Transporters [IRT]), under such condition. In this study, cDNA amplified fragment length polymorphism (cDNAAFLP) analysis was employed to identify genes differentially expressed at the root apex level following Fe deficiency treatment application. The expression patterns of the most interesting transcript derived fragments (TDFs) were validated through semiquantitative RT-PCR analysis. This study allowed us to identify a set of new cucumber genes overexpressed under Fe deficiency, such as those coding for calmodulin, SNAP, TIM23 and VPPase. Furthermore, we also observed that calmodulin protein accumulated in Fe-deficient root apices. This last result allows us to hypothesise that a signal transduction cascade might be involved in the transduction of the Fe deficiency signal or, more likely, it may be involved in the induction and regulation of the metabolic changes induced by Fe deficiency
\end{abstract}

Additional Key words: calmodulin, Cucumis sativus L., iron deficiency, semiquantitative RT-PCR (sqRT-PCR), Strategy I, transcript-derived fragments (TDFs), transcriptomic analysis.

Abbreviations: AFLP, amplified fragment length polymorphism; BPDS, bathophenantrolinedisulfonate; PVDF, Polyvinylidene Difluoride; TDF, transcript derived fragments

\title{
Acknowledgements
}

The authors wish to thank Dr. H. Hartings for critical reading of the manuscript. The research was financially supported by a grant from Ministero dell'Istruzione, dell'Università e della Ricerca (MiUR). We thank The Institute of Vegetables and Flowers, Chinese Academy of Agricultural Sciences (IVF-CAAS) for the release of information about the cucurbit genome. The Authors thanks the anonymous Reviewers for the valuable comments useful to improve the manuscript.

* Author for correspondence : phone: +00390250316522;fax: +00390250316521e-mail: gianpiero.vigani@unimi.it

footnote: a these authors equally contributed to this work 


\section{Introduction}

Iron exists, in well aerated soils, mainly as scarcely soluble oxides and oxi-hydroxides and therefore is not freely available to plants uptake, notwithstanding its abundance. In plants, Fe uptake and homeostasis are tightly regulated to ensure both a sufficient supply of Fe from the soil and the avoidance of a toxic excess in the cell. Iron deficiency induces various responses at the root level, aimed to increase the availability of the ion in the rhizosphere for plant uptake. Strategy I plants (dicotyledonous and non-graminaceous plants) are able to respond to a lack of Fe in the soil by increasing i) the Fe reduction capacity of root tissues, ii) the acidification of the rhizosphere to increase Fe solubility and iii) the uptake activities in rhizodermal root cells (i.e. Fe(III)-chelate reductase $[\mathrm{FC}-\mathrm{R}], \mathrm{H}^{+}-\mathrm{ATPase}$ and Iron Regulated Transporters [IRT], respectively) (Curie and Briat 2003). The increase in FC-R and $\mathrm{H}^{+}$-ATPase activities needs a continuous supply of energetic substrates to keep the system working. This effort needs a reorganization of metabolic pathways to efficiently sustain activities linked to the acquisition of iron; in fact, the energetic metabolism of carbohydrates has been shown to be involved in these responses (Zocchi 2006, Vigani and Zocchi 2009).

14 Thus, it is evident that the effects of Fe deficiency on plant metabolism are multifaceted, and that more information is required to increase our comprehension of the regulatory mechanisms activated by plants under this condition.

Proteome and transcriptome analyses are the most useful tools to obtain a wide collection of information about the metabolic status of a particular tissue and under particular conditions. For Strategy I plants, some studies on proteomic investigations under Fe deficiency (Li et al. 2008; Donnini et al. 2010), as well as some studies showing a microarray analysis of differentially expressed transcript profiles in Fe-deficient Arabidopsis thaliana

21 (Thimm et al. 2001; Buckhout et al. 2009) and soybean (Glycine max (L.) Merr.) plants (O'Rourke et al. 2007) have recently been published. Transcript profiling techniques allow the simultaneous examination of gene expression under different 24 experimental conditions. The PCR-based technique of cDNA-amplified fragment length polymorphism (cDNAAFLP) is widely available at a low cost, even if for various plant species there is little information at the molecular level (Breyne and Zabeau 2001). The sensitivity and specificity of the method allows the detection of even poorly expressed genes and permits us to distinguish between homologous sequences (Breyne et al. 2003; Fukumura et al. 2003). This is an extremely efficient labor-intensive mRNA fingerprinting method for the isolation of those genes which show differential expression in stressed conditions. Moreover this technique enables the discovery of genes that have not been previously identified or predicted from sequence analysis. 
However, it should be noted that molecular components that are controlled by post-transcriptional regulation or by post-translational modification cannot be identified.

3 The AFLP technology has been used predominantly for assessing the degree of variability among plant cultivars, establishing linkage groups in crosses and saturating genomic regions with markers for gene landing efforts. The AFLP fragments may also be used as physical markers to determine the overlap and positions of genomic clones and to integrate genetic and physical maps (Vuylsteke et al. 2007 and references therein, Beharav et al. 2010, Kang et al. 2010, Sikdar et al. 2010, Cuesta et al, 2010 ).

Keeping the above in view, the objective of our study was to identify genes that were differentially expressed in response to Fe deficiency in cucumber (Cucumis sativus L.). Cucumber can be considered a model Strategy I plant since it exhibits intense responses to Fe deficiency compared to other species (e.g. strong rhizosphere acidification). While the responses of cucumber plants to Fe deficiency have been largely characterized only at the biochemical level, the studies of Fe-deficient Arabidopsis thaliana plants are mainly performed at molecular level, lacking a satisfactory biochemical characterization (Curie and Briat, 2003). In this study, cDNA amplified fragment length polymorphism (cDNA-AFLP) analysis was employed to identify genes which showed differential expression under Fe deficiency treatment at the root apex level in cucumber roots. The expression patterns of the some TDFs were validated through semiquantitative RT-PCR analysis. Several differentially expressed cDNA fragments were isolated and sequenced, and their possible functions are discussed.

\section{Materials and methods}

Plant material and growth conditions: Cucumber plants (Cucumis sativus L. cv. Marketmore 76) were grown in a nutrient solution as reported by Vigani et al. (2009). Seven-day-old plants grown in a complete nutrient solution were divided, one half was transferred to the same nutrient solution and the other half to a nutrient solution without Fe. Sampling was performed 7 days after the transfer, and 4 hours after light onset.

In vivo localisation of the reduction capacities: Visualization and localization of Fe(III) reduction was performed by embedding the roots in a agar medium as described in Marschner et al. (1982) in the presence of the bathophenantrolinedisulfonate (BPDS) reagent which forms a stable, water soluble, red complex with $\mathrm{Fe}^{2+}$ and only a weak complex with $\mathrm{Fe}^{3+}$.

cDNA Preparation and AFLP Analysis: Total RNA was isolated from 100-200 $\mathrm{mg}$ of root apexes $(0.5-1 \mathrm{~cm}$ from the tip) using TRIzol reagent (Sigma Aldrich, Milano Italy) according to the manufacturer's instructions. Poly A ${ }^{+}$ RNA was isolated following the Oligotex mRNA Spin-column protocol (Qiagen, Milano Italy). The concentration 
of Poly $\mathrm{A}^{+}$RNA was checked spectrophotometrically at $260 \mathrm{~nm}$ and about $500 \mathrm{ng}$ of Poly $\mathrm{A}^{+}$RNA from each sample was used for cDNA preparation. First strand cDNA was synthesized following the reverse transcriptase SuperScript protocol (Invitrogen, Milano Italy). Contaminating genome DNA was removed by DNaseI treatment (Amersham Biosciences, Uppsala, Sweden). The cDNA-AFLP analysis was performed according to Bachem (1996) as modified by Hartings (1999) (see Table I in the supplementary analytical procedures; for more detail refer to the website http://www.diprove.unimi.it/info/msg1.htm or contact the corresponding author). The validation of all the steps of the experiment have been done with three independent biological replicates and each of them has been technically repeated twice.

The eluted TDFs were cloned into the pBlueScript II plasmid (Stratagene, Cedar Creek, Texas) according to the manufacturer's instructions. The recombinant plasmids were purified using QIAprep Spin Miniprep Kit (Qiagen, Milano Italy) following the manufacturer's protocol. Resulting sequences were then analysed by Primm (Primm s.r.l., Milano, Italy). After removal of vector sequences, database search was performed. The nucleotide as well as translated protein sequences were analyzed for their homology using the publicly available Nucleotide collection of the Cucurbit genome database (http://www.icugi.org/cgi-bin/ICuGI/tool/blast.cgi) using Cucumber database (v2.0) and BLASTN program. For further information about the AFLP procedure and used primers contact the corresponding author or visit the http://www.diprove.unimi.it/info/msg1.htm.

Semiquantitative RT-PCR analysis: Root tissues were pulverised in liquid nitrogen using a mortar and pestle, total RNA was extracted using Trizol ${ }^{\circledR}$ reagent (Invitrogen, Milano Italy) and first strand cDNA synthesis was carried out using the iScript ${ }^{\mathrm{TM}} \mathrm{CDNA}$ Synthesis Kit (Bio-Rad, Milano, Italy) according to the manufacturer's instructions. Actin, which is a gene constitutively expressed, was used as an internal control. Semiquantitative RT-PCR analysis of Strategy I genes (Fig 1C) and of TDFs was performed according to Donnini et al (2010). The relative expression level of the TDFs (Supplemental Figure 1) was determined es reported in supplental analytical procedures. The primers used for semiquantitave RT-PCR were designed from the selected sequences of the TDFs using the FAST-PCR Version (Supplemental Table 2).

Protein gel blot analysis: Soluble proteins extracted from roots of plants grown in the presence and in the absence of Fe were loaded onto a discontinuous SDS-polyacrylamide gel (according to De Nisi and Zocchi 2000). After SDS-PAGE, proteins were electrophoretically transferred to Polyvinylidene Difluoride (PVDF) membrane filters (Sigma) and the immunoreaction with Monoclonal Anti-Calmodulin antiserum (Sigma) was performed according to the manufacturer's instructions. The detection procedure was performed by ExtraAvidin 
peroxidase Staining Kit Mouse (Sigma) according to the manufacturer's instructions. Protein concentration was determined according to Bradford (1976).

\section{Results and Discussion}

We focused our analysis on the root apex only, since it is the main site displaying ion uptake in which Strategy I activities are strongly induced (Landsberg 1986, 1994). Cucumber plant displayed notable morphological changes at the root apex level (Fig.1A) and showed pronounced in vivo Fe reduction (Fig.1B) compared to the control. Furthermore, the genes encoding the enzymes typically induced under Fe-deficiency induced (FC-R, $\mathrm{H}^{+}-$ ATPase and IRT) were over-expressed in -Fe root apex compared with the control (Fig. 1C). Surprisingly, TDFs homologous to FC-R, $\mathrm{H}^{+}$-ATPase and IRT were not detected in Fe-deficient roots, nor were other typical metabolic markers related to Strategy I response (e.g. phosphoenolpyruvate carboxylase). The lack of detection of these TDFs could be explained by taking into account the possible limitations inherent in the cDNA-AFLP methodology: i) the primers used may have been unable to amplify the transcripts relative to Strategy I genes; ii) the under-representation of transcripts lacking a recognition site for the restriction enzymes used; iii) the cDNA-AFLP technology is based on a double digest, short fragments may be generated and subsequently lost in the analysis; iv) the missing TDFs could have a sequence length under $100 \mathrm{bp}$ or above 500 bp, which was the range considered in cDNA-AFLP analysis. About 2000 fragments ranging from 100 to $500 \mathrm{bp}$ in length were typically observed on the gels examined. We identified 26 differentially expressed TDFs (Table

19 1). These TDFs were eluted from the gel, re-amplified, and cloned. Twenty-two (85\%) out of 26 TDFs were up regulated and 4 (15\%) were down regulated under Fe deficiency.

21 An investigation in the Cucurbit genome database revealed a relationship between the selected 26 clones and the 22 genes involved in environmental stress. The biological role of some of these cloned genes can be inferred from sequence similarity to previously studied plant proteins. Among the 22 up-regulated TDFs, the comparison

24 between the homologues of these sequences and those in the database suggested that most of them are involved in processes such as protein synthesis (e.g. TDFs \# 5, 6 and 19), in the respiration pathway (e.g. TDFs \# 7, 8 and 26 9), ion transport, compartmentalization and cellular homeostasis (e.g. TDFs \# 10 and 12), cell signalling (e.g. TDF \# 14) and cell division (e.g. TDFs \# 15, 16 and 20). The remaining TDFs were not found in the cucumber database

28 (e.g.TDFs \# 2 and 17, 18, 21, 22, 25). In Table 1 the TDFs homologies are reported. Out of the 26 clones, we 
to validate our cDNA-AFLP data through semiquantitative RT-PCR analysis according to methods suggested by several authors (Bachem et al. 1996, Ditt et al. 2001, Sojikula et al. 2010).

\section{TDFs with putative function in mitochondrial respiration}

5 The TDF \#7 was homologous to TIM23 gene (AT1G7530.1) from Arabidopsis thaliana (Table 1) encoding for a translocase which represents the main entry site for proteins addressed to the matrix and the inner mitochondrial membrane (Bauer et al. 2000; Koehler 2000; Jensen et al. 2002; Endo et al. 2003; Rehling et al. 2003).

The TDF identified by cDNA-AFLP (Fig. 2) and confirmed by sqRT-PCR (Fig. 3A, supplemental Fig.1) showed an overexpression in Fe-deficient root apices which suggests an increase in the mitochondrial protein import under Fe deficiency. Despite the recent observation that the content of many proteins decreases in mitochondria of Fedeficient cucumber (Vigani et al. 2009), it has been hypothesized that mitochondria still play a pivotal role in metabolic changes occurring under Fe deficiency through the activation of alternative metabolic pathways (i.e. alternative NAD(P)H dehydrogenase and metabolite shuttles) (Vigani and Zocchi 2009, 2010). In agreement with our results, Lister et al. (2004) suggest that the transcription of mitochondrial import component genes is induced when mitochondrial function is limited. In fact, Arabidopsis thaliana cells treated with rotenone and antimycin A showed a strongly induced expression of mitochondrial import component genes, TIM23 among them. Since TIM23 is specifically involved in the protein transport into the matrix, its induction suggests an enhancement of protein request in the matrix. In fact, it is well documented that some activities belonging to the Krebs cycle (López-Millan et al. 2000) and the demand for related proteins (Li et al. 2008) increase under such 21 stress condition. TDF \#8 was homologous to AT4G02580 gene from Arabidopsis thaliana (Tab. 1) encoding for a $24 \mathrm{kDa}$ subunit of mitochondrial complex I [NADH dehydrogenase (ubiquinone)]. The TDF identified by cDNA-AFLP (Fig. 2) and confirmed by sqRT-PCR (Fig. 3A, supplemental Fig.1) showed an overexpression in Fe-deficient root apices. Thimm et al. (2001) observed a transcript overexpression of a NADH oxidoreductase, suggesting a transcriptional induction of a gene encoding for a complex I subunit. However we previously observed an almost undetectable protein band of NAD9 (a Complex I subunit) while complex I activity was strongly decreased (Vigani et al. 2009). The contrasting data between transcriptional induction and activity inhibition of complex I could be explained in two ways: i) the presence of different transcriptional and translational regulations, ii) transcript induction observed in plants grown first in the presence of Fe and then subjected to Fe deprivation, 
while the protein content and complex I activity determined on 10-day-old cucumber plants grown directly, after germination, in the absence of Fe.

\section{TDFs with putative function in the proton and vesicle transport}

TDF \#10 was homologous to the AVP2 gene (AT1G78920) from Arabidopsis thaliana, encoding for a vacuolarPPase (V-PPase) (Table 1). The TDF identified by cDNA-AFLP (Fig. 2) and confirmed by sqRT-PCR (Fig. 3A, supplemental Fig.1), showed an overexpression in Fe-deficient root apices. Among the proton pumps, the plant cells possess a vacuolar $\mathrm{H}^{+}$-PPase (V-PPase), which is able to acidify the vacuolar lumen by hydrolysing PPi (Maeshima 2000). The overexpression of the TDF \#10 in cucumber root apices suggests an induction of vacuolar lumen acidification under Fe deficiency. On the contrary, Espen et al. (2000) showed a vacuolar pH increase in

11 Fe-deficient cucumber roots. However, it has been shown that the V-PPase is not restricted only to the tonoplast but it is also present and active in the plasmalemma (PM), in the trans-Golgi network (TGN) and in multivesicular bodies (Ratajczak et al. 1999). Thus, the transcript induction of a V-PPase in cucumber root apices could be also related to the vesicular traffic in the cell and not only to its tonoplast activity. In fact, we have also identified a gene over-expressed in this condition (TDF \#26, Fig. 2, Fig. 3 and supplemental Fig. 1), homologous to AT3G56190 gene from Arabidopsis thaliana coding for $\alpha$-SNAP (Soluble NSF Attachment Protein) (Table 1). SNAP proteins belong to the SNARE (soluble N-ethylmaleimide sensitive factor attachment protein receptors) protein family, which are involved in a specific vesicles fusion process during vesicular transport (Uemura et al. 2004). Vesicles are used to transport molecules, for instance proteins, to specific locations. The docking of the vesicle to the target membrane, causes the recruitment of two general soluble trafficking factors, NSF (Nethylmaleimide-Sensitive Factor) and $\alpha$-SNAP leading to membrane fusion and delivery of the contents of the transport vesicle (Fairman and Wagner 2003). This finding sheds new light on the link between exocytosis processes and Fe deficiency responses occurring in roots, mainly for two reasons. The first one could be related

24 to the formation of transfer cells - a process well documented in the Fe-deficient root tissues (Landsberg 1994), but not yet completely understood. We hypothesize that an enhancement of vesicle trafficking towards to the PM increases the apposition of organic compounds for cell wall synthesis. The second reason could be related to the over-expression of the V-PPase gene observed in this study. We hypothesize that Fe deficiency can promote exocytosis processes in the cell, enhancing vesicular traffic from the cytosol to the PM. The vesicles formed could be characterized by the presence of V-PPase which could acidify the vacuolar lumen. Once the vesicles containing protons reach the PM the fusion of these membranes could lead at the same time to a) the apoplastic 
acidification assisting the $\mathrm{PM} \mathrm{H}^{+}-\mathrm{ATPase}$ activity and b) the proton extrusion into the apoplast using the energy from PPi hydrolysis avoiding the consumption of ATP, which in this condition is a metabolic limiting factor.

\section{TDF \#14 putatively encode calmodulin}

TDF \#14 was homologous to CAM7 gene (AT3G438101) from Arabidopsis thaliana encoding for calmodulin

(Table 1). Calmodulin (CaM) is a conserved multifunctional calcium sensor that mediates intracellular $\mathrm{Ca}^{2+}$

signalling and regulates diverse cellular processes by interacting with calmodulin-binding proteins (Ikura and Ames 2006; Bouche et al. 2005). The over-expression of the transcript encoding for calmodulin in Fe-deficient root apexes has been observed as a polymorphic band in cDNA-AFLP (Fig. 2) and confirmed by sqRT-PCR analysis (Fig. 3A, supplemental Fig.1). Moreover, there are no papers about CaM induction under the condition of Fe deficiency. This finding is not unexpected since new stimuli were provided by changing the growing conditions. This finding might suggest a direct involvement of CaM in the transduction of the Fe deficiency signal or, more likely, its involvement in the induction and regulation of the metabolic changes which accompany the responses induced by Fe deficiency; of course we are aware that more results are required to finally corroborate this hypothesis. Interestingly, the Fig. 3B showed that the transcript strongly increased about 1 day after the induction of the Fe deficiency condition, while the protein weakly accumulated after 1 and 3 days and just after 5 days of the removal of the Fe from the nutrient solution the CaM protein accumulated strongly. Moreover, some authors observed a transcript induction of genes encoding for 14-3-3 proteins and some protein kinases in Fedeficient Arabidopsis thaliana roots which should be target proteins of the CaM-transduced signal (Colangelo and Guerinot. 2004, Buckhout et al. 2009).

We aware that the identification of the precise role of these genes and their specific involvement under Fe deficiency needs further investigation.

\section{References}

Bachem, C.W.B., van der Hoeven, R.S., de Brujin, S.M., Vreugdenhil, D., Zabeau, M., Visser, R.G.F.: Visualization of differential gene expression using a novel method of RNA fingerprinting based on AFLP: analysis of gene expression during potato tuber development. - Plant J. 9: 745-753, 1996.

Bauer, M.F., Hofmann, S., Neupert, W., Brunner, M.: Protein translocation into mitochondria: the role of TIM complexes. - Trends Cell. Biol. 10: 25-31, 2000.

Beharav, A., Maras, M., Kitner, M., Šuštar-Vozlič, J., Sun, G.L., Doležalová, I., Lebeda, A., Meglič, V.: Comparison of three genetic similarity coefficients based on dominant markers from predominantly self-pollinating species. Biol. Plant. 54: 54-60, 2010.

Bouche, N., Yellin, A., Snedden, W.A., Fromm, H.: Plant-specific calmodulin-binding proteins. - Annu. Rev. Plant Biol. 56, 435-466, 2005. 
Bradford, M.M.: A rapid and sensitive method for the quantization of micrograms quantities of protein utilizing the principle of protein-dye binding. - Anal. Biochem. 72: 248-254, 1976.

Breyne, P., Dreesen, R., Cannoot, B., Rombaut, D., Vandepoele, K., Rombauts, S., Vanderhaeghen, R., Inzé, D., Zabeau, M.: Quantitative cDNA-AFLP analysis for genome-wide expression studies. - Mol. Gen. Genom. 269: 173-179, 2003.

Breyne, P., Zabeau, M.: Genome-wide expression analysis of plant cell cycle modulated genes. - Curr. Opin. Plant Biol. 4: 136-142, 2001.

Buckhout, T.J., Yang, T.J., Schmidt, W.: Early iron-deficiency-induced transcriptional changes in Arabidopsis roots as revealed by microarray analyses. - BMC Genomics 10: 147, 2009.

Colangelo, E.P., Guerinot, M.L.: The essential basic helix-loop-helix protein FIT1 is required for Fe deficiency response.- Plant Cell 16: 3400-3412, 2004.

Cuesta, C., Ordás, R.J., Rodríguez, A. Fernández, B.: PCR-based molecular markers for assessment of somaclonal variation in Pinus pinea clones micropropagated in vitro. - Biol. Plant. 54: 435-442, 2010.

Curie, C., Briat, J.F.: Iron transport and signalling in plants. Annu. Rev. Plant Biol. 54: 183-206, 2003.

De Nisi, P., Zocchi, G.: Phosphoenolpyruvate carboxylase in cucumber (Cucumis sativus L.) roots under iron deficiency: activity and kinetic charaterisation. - J. Exp. Bot. 51: 1903-1909, 2000.

Ditt, R.F., Nester, E.W., Comai, L.: Plant gene expression response to Agrobacterium tumefaciens. - Proc. Natl. Acad. Sci. USA. 98: 10954-10959, 2001.

Donnini, S., Prinsi, B., Negri, A.S., Vigani, G., Espen, L., Zocchi, G.: Proteomic characterization of iron deficiency response in Cucumis sativus L. roots. - BMC Plant Biol. 10: 268, 2010.

Endo, T., Yamamoto, H., Esaki, M.: Functional cooperation and separation of translocators in protein import into mitochondria, the double-membrane bounded organelles. - J. Cell. Sci. 116: 3259-3267, 2003.

Espen, L., Dell'Orto, M., De Nisi, P., Zocchi, G.: Metabolic responses in cucumber (Cucumis sativus L.) root under Fe-deficiency: a 31NMR in vivo study. - Planta. 210: 985-992, 2000.

Fairman, R., Wagner, J.: Structural studies of $\alpha$-SNAP. - Biology 300a Quarter 1 Biochemistry of Proteins \& Nucleic Acids 2003.

Fukumura, R., Takahashi, H., Saito, T., Tsutsumi, T., Fujimori, A., Sato, S., Tatsumi, K., Araki, R., Abe, M.: A sensitive transcriptome analysis method that can detect unknown transcripts. - Nucl. Ac. Res. 31: 94, 2003.

Hartings, H.: High resolution fingerprinting of transcribed genes by means of a modified cDNA-AFLP method. Short Communications. - Maydica. 44: 179-181, 1999.

Ikura, M., Ames, J.B.: Genetic polymorphism and protein conformational plasticity in the calmodulin superfamily: two ways to promote multifunctionality. - Proc. Natl. Acad. Sci. USA. 103:1159-1164, 2006.

Jensen, R., Dunn, C.: Protein import into and across the mitochondrial inner membrane: role of the TIM23 and TIM22 translocation. - Biochim. Biophys. Acta. 1592: 25-34, 2002.

Kang, S.-Y., Lee, K.J., Lee, G.-J., Kim, J.-B., Chung, S.-J., Song, J.Y., Lee, B.-M., Kim, D.S.: Development of AFLP and STS markers linked to a waterlogging tolerance in Korean soybean landraces. - Biol. Plant. 54: 61-68, 2010.

Koehler, C.M.: Protein translocation pathways of the mitochondrion. - FEBS Lett. 476: 27-31, 2000.

Landsberg, E.C.: Function of rhizodermal transfer cells in the Fe stress response mechanism of Capsicum annuum L. - Plant Physiol. 82: 511-517, 1986.

Landsberg, E.C.: Transfer cell formation in sugar beet roots induced by latent Fe deficiency. - Plant Soil. 165: 197-205, 1994.

Li, J., Wu, X.D., Hao, S.T., Wang, X.J., Ling, H.Q.: Proteomic response to iron deficiency in tomato root. -Proteomics. 8: 2299-311, 2008.

Lister, R., Chew, O., Lee, M.N., Heazlewood, J.L., Clifton, R., Parker, K., Millar, A.H., Whelan, J.: A transcriptomic and proteomic characterization of the arabidopsis mitochondrial protein import apparatus and its response to mitochondrial dysfunction. - Plant Physiol. 134: 777-789, 2004.

López-Millán, A.F., Morales, F., Andaluz, S., Gogorcena, Y., Abadía, A., De Las Rivas, J., Abadía, J.: Responses of sugar beet roots to iron deficiency. Changes in carbon assimilation and oxygen use. - Plant Physiol. 124: 885897, 2000.

Maeshima, M.: Vacuolar H+-pyrophosphatase. - Biochim. Biophys. Acta. 1465: 37-51, 2000.

Marschner, H., Römheld, V., Ossenberg-Neuhaus, H.: Rapid method for measuring changes in $\mathrm{pH}$ and reducing process along roots of intact plants. - Zeit. Pflanz. Boden. 105: 407-416, 1982.

O'Rourke, J.A., Charlson, D.V., Gonzalez, D.O., Vodkin, L.O., Graham, M.A., Cianzio, S.R., Grusak, M.A., Shoemaker, R.C.: Microarray analysis of iron deficiency chlorosis in near-isogenic soybean lines. - BMC Genomics. 8: 476, 2007.

Ratajczak, R., Hinz, G., Robinson, D.G.: Localization of pyrophosphatase in membranes of cauliflower inflorescence cells. - Planta. 208: 205-211, 1999.

Rehling, P., Pfanner, N., Meisinger, C.: Insertion of hydrophobic membrane proteins into the inner mitochondrial membrane-a guided tour. - J.Mol.Biol. 326: 639-657, 2003. 
Sikdar, B., Bhattacharya, M., Mukherjee, A., Banerjee, A., Ghosh, E., Ghosh, B., Roy, S.C.: Genetic diversity in important members of Cucurbitaceae using isozyme, RAPD and ISSR markers. - Biol. Plant. 54: 135-140, 2010.

Sojikula, P., Kongsawadworakula, P., Viboonjuna, U., Thaiprasitc, J., Intawong, B., Narangajavanaa, J., Svastid, M.R.J.: AFLP-based transcript profiling for cassava genome-wide expression analysis in the onset of storage root formation. - Physiol. Plant. 140: 198-198, 2010

Thimm, O., Essigmann, B., Kloska, S., Altmann, T., Buckhout, T.J.: Response of arabidopsis to iron deficiency stress as revealed by microarray analysis. - Plant Physiol. 127: 1030-1043, 2001.

Uemura, T., Ueda, T., Ohniwa, R.L., Nakano, A., Takeyasu, K., Sato, M.H.: Systematic analysis of SNARE molecules in Arabidopsis: dissection of the post-Golgi network in plant cells. - Cell Struct. Funct. 29: 49-65, 2004.

Vigani, G., and Zocchi, G.: Effect of Fe deficiency on mitochondrial alternative NAD(P)H dehydrogenases in cucumber roots. - J. P. Physiol. 167: 666-669.

Vigani, G., Maffi, D., Zocchi, G.: Iron availability affects the function of mitochondria in cucumber roots. - New Phytol. 182: 127-136, 2009.

Vigani, G., Zocchi, G.: The fate and the role of mitochondria in Fe deficient roots of Strategy I plants. - Plant Signal. Behav. 4: 375-379, 2009.

Vuylsteke, M., Peleman, J.D., van Eijk, M.J.T.: AFLP-based transcript profiling (cDNA-AFLP) for genome-wide expression analysis. - Nat. Prot. 2: 1399-1413, 2007.

Waters, B.M., Lucena, C., Romera, F.J., Jester, G.G., Wynn, A.N., Rojas, C.L., Alcantara, E., Perez-Vicente, R.: Ethylene involvement in the regulation of the $\mathrm{H}^{+}$-ATPase CsHA1 gene and of the new isolated feric reductase CsFR01 and iron transporter CsIRT1 genes in cucumber plants. - Plant Physiol. Biochem. 45: 293-301, 2007.

Zocchi, G.: Metabolic changes in iron-stressed dicotyledonous plants. In: Barton, L.L., Abadía, J., (ed.): Iron Nutrition in Plants and Rhizospheric Microorganisms. Pp 359-370. Dordrecht - The Netherlands - Springer 2006. 